\title{
Kernos
}

Revue internationale et pluridisciplinaire de religion grecque antique

$23 \mid 2010$

Varia

\section{Maria Eugenia DE LA NUEZ, Les cultes d'Athéna en Asie mineure}

\section{Stéphanie Paul}

\section{OpenEdition \\ Journals}

\section{Édition électronique}

URL : http://journals.openedition.org/kernos/1629

DOI : 10.4000/kernos. 1629

ISSN : 2034-7871

\section{Éditeur}

Centre international d'étude de la religion grecque antique

\section{Édition imprimée}

Date de publication : 1 janvier 2010

Pagination : $382-384$

ISSN : 0776-3824

Référence électronique

Stéphanie Paul, «Maria Eugenia de LA NuEz, Les cultes d'Athéna en Asie mineure », Kernos [En ligne], 23 | 2010, mis en ligne le 15 septembre 2011, consulté le 21 septembre 2020. URL : http:// journals.openedition.org/kernos/1629; DOI : https://doi.org/10.4000/kernos.1629

Ce document a été généré automatiquement le 21 septembre 2020.

Kernos 


\title{
Maria Eugenia DE LA NUEZ, Les cultes d'Athéna en Asie mineure
}

\author{
Stéphanie Paul
}

\section{RÉFÉRENCE}

Maria Eugenia DE LA NUEZ, Les cultes d'Athéna en Asie mineure, Messina, Di.Sc.A.M. -

DiSTAMU, 2009. 1 vol. $17 \times 24,5$ cm, 167 p. (Polifemo supplemento, 6). ISBN : 88-8268-018-5.

1 Version remaniée d'une thèse soutenue fin 2005 à l'Université de Madrid, en cotutelle avec l'Université de Bordeaux 3 Ausonius, ce petit volume propose ce qui promet d'être une synthèse sur la figure d'Athéna et sur ses cultes en Asie mineure, y compris les îles qui en sont proches. Comme elle l'annonce dès son introduction, l'intérêt de l'A. porte principalement sur l'étude de la déesse au sein de la polis et des rapports qu'elle entretient avec celle-ci, qui constitue l'un de ses champs d'action privilégiés. La fourchette chronologique des témoignages envisagés est particulièrement large, puisqu'elle s'étend du VIII ${ }^{\mathrm{e}}$ s. av. J.-C. au IV e ap. J.-C.

2 La matière est articulée en deux parties principales, qui ont pour objet de traiter respectivement des «fonctions d'Athéna ", puis de la "réalité des cultes». Dans la première partie, l'A. vise donc à dessiner un portrait de la déesse dans l'Asie mineure au moyen des traditions mythiques qui y sont localisées (Chapitre I) d'une part, et des différents domaines dans lesquels elle exerce son activité (Chapitre II) d'autre part. Dans le chapitre I, qui présente les mythes selon une démarche régionale, elle met en évidence, en plus du caractère poliade d'Athéna, son rapport avec la fécondité et la fertilité. Dans le chapitre II, l'A. s'intéresse aux épiclèses de la déesse qui renvoient à ses différents domaines d'activités. Elle classe ces derniers en trois catégories : 1) le rapport à " la vie ", où elle regroupe les thèmes de la fécondité/fertilité, la protection des activités féminines et la courotrophie; 2) la protection de la communauté, à savoir Athéna dans son rôle de patronne de la polis et de ses citoyens; 3) le rapport à «la mort », le caractère "chthonien » et « apotropaïque » de la déesse, sa protection sur le 
passage vers l'au-delà. Cette partie se conclut par le développement de deux exemples qui sont ceux de Troie et de Pergame, deux cités où, selon l'A., le culte d'Athéna revêtait un caractère double.

3 Après ce parcours de la figure de la déesse, l'A. entend confronter ses conclusions aux « réalités des cultes ». Dans cette deuxième partie, elle fait donc preuve d'une volonté de présenter les données d'une manière plus systématique, en adoptant cette fois une approche thématique. Le chapitre IV traite de l'organisation spatiale du culte, l'emplacement des sanctuaires et leur configuration -, et mène à la conclusion que, si la situation des lieux de culte varie en fonction de la cité, ils correspondent généralement à une typologie grecque régulière. Dans le chapitre $\mathrm{V}$, c'est l'organisation matérielle du culte qui est envisagée, à savoir son financement et le personnel. Enfin, le dernier chapitre regroupe les fêtes, les sacrifices et les offrandes. Les deux premiers points sont distingués, parfois à tort. En effet, certains sacrifices ne manquent pas de s'intégrer dans une fête de plus grande envergure, même si le caractère de la documentation épigraphique n'en conserve pas toujours le nom ou une description complète.

La méthode adoptée et l'ordonnancement de la matière pourront étonner le lecteur. Dans la mesure où le sujet de l'étude est déterminé par des critères géographiques, on aurait pu s'attendre à retrouver cette approche régionale au sein même de l'exposé, qui aurait ensuite abouti à une synthèse. L'A. procède, en quelque sorte, de manière inverse : elle tente de dresser un portrait de la déesse en se fondant sur les traditions mythiques et les épiclèses qui la définissent afin de la confronter à «la réalité des cultes ». Cette manière de procéder a l'avantage d'éviter une présentation des données peut-être un peu longue et fastidieuse, mais qui aurait pu fournir un outil de travail précieux pour qui s'intéresse à la vie religieuse de l'Asie mineure. Ce choix présente également un certain nombre d'inconvénients. Il mène à l'éclatement des données, ou au contraire, à des redondances, ce qui nuit parfois à la clarté de l'exposé, étant donné l'absence de renvois internes. Ce problème aurait pu être résolu en partie par la mise à disposition d'index qui fait malheureusement défaut. Le lecteur éprouvera à l'occasion des difficultés à apprécier pleinement l'argumentation sans avoir en main toutes les pièces du dossier. Un autre problème plus spécifique lié à la répartition de la matière est la dissociation de l'étude des épiclèses, qui trouve sa place dans la première partie, de celle des cultes, qui fait l'objet de la seconde. En effet, les épiclèses s'inscrivant dans un contexte cultuel précis, on peut difficilement envisager leur étude sans tenir compte de celui-ci.

Une même absence de remise en contexte, dont l'importance est pourtant soulignée à diverses reprises par l'A. (par exemple p. 16, 146, etc.), s'observe à d'autres endroits de l'étude. Une illustration en est la présentation des offrandes par ordre chronologique (p. 151-160), en faisant abstraction de tout contexte archéologique. Or, on sait à quel point la remise en perspective de ce type de témoignage est cruciale pour son interprétation ${ }^{1}$. Même si l'on peut apprécier l'effort de présenter les données d'une manière plus systématique dans sa deuxième partie, le fait de n'avoir pas replacé les témoignages dans leur contexte local ne nous permet pas, par exemple, de percevoir les relations entre différents cultes d'Athéna au sein d'une même cité, ou encore de la situer au sein d'un panthéon local.

6 À cela s'ajoute un certain manque de rigueur dans le traitement de la documentation. Un exemple révélateur, qui m'est plus familier en raison de mes propres recherches ${ }^{2}$, en est le commentaire proposé p. 145 d'une inscription de Cos concernant la fondation 
de fêtes en l'honneur de Zeus Sôter et d'Athéna Sôteira par un certain Pythoclès (ICos ED 82). Cette inscription est très lacunaire $( \pm 18$ lettres conservées par ligne pour plus du double restitué) et son interprétation se fonde par conséquent en grande partie sur des restitutions, ce qui n'est pas mentionné. Le point qui intéresse l'A. est le financement prétendument "particulier» de ces fêtes: «le "roi" donne aux trésoriers l'argent nécessaire aux besoins de la fête et ceux-ci le donnent à leur tour au personnel chargé de l'organisation ». Et de préciser un peu plus loin que « le "roi" était l'archonte basileos (sic) qui, dans certaines cités comme à Athènes, avait le soin des activités religieuses ". Or, l'archonte-basileus n'est pas attesté à Cos. Le seul mot qui pourrait avoir été

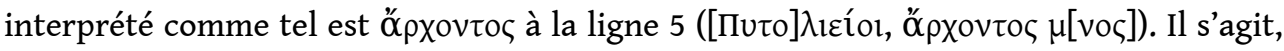
soit d'une datation par le nom de l'archonte - mais l'absence de la préposition غ̇đí ou d'un nom au génitif infirme cette possibilité -, soit, si la restitution $\mu \eta v o ́ \varsigma$ est correcte, d'une expression signifiant "à partir du mois untel », comme on la trouve dans d'autres inscriptions ${ }^{3}$. Le texte ne témoigne donc nullement d'une éventuelle procédure inhabituelle. Au contraire, si l'on se fie aux restitutions, on lit que les tamiai donnent l'argent à ceux qui doivent accomplir les sacrifices, selon une pratique bien connue. Il n'y a pas davantage de raison de supposer l'existence de trésoriers « d'Athéna », comme il est écrit p. 126 en se fondant sur cette même inscription. Les tamiai, qui n'apparaissent que dans les restitutions, seraient ici de toute évidence ceux de la cité, et ne sont pas attachés à un culte particulier. De telles inexactitudes me sont apparues à plusieurs reprises lorsqu'il était question des cultes de Cos. En ce qui concerne les régions qui me sont moins familières, je laisserai le lecteur compétent seul juge de la pertinence des interprétations proposées, mais les faiblesses soulignées invitent à la prudence.

7 L'ouvrage pâtit encore de l'absence d'une bibliographie finale. Les notes de bas de page sont parfois trop peu fournies, les ouvrages de référence n'apparaissent pas toujours là où on les attendrait. Les sources antiques ne sont pas toujours citées, ou parfois de manière erronée (ex. p. 134 n. 17 : lire ED 82 et non EV 82; p. 52, n. 74: LSCG, 258 ne correspond à rien) et l'A. renvoie fréquemment aux opinions des savants modernes sans préciser de références (p. 21, 28, 51, 140, etc.). Par ailleurs, dans ses interprétations, elle recourt fréquemment à des concepts très discutés dans la recherche actuelle, comme la distinction olympien/chthonien, la notion de fertilité/ fécondité, de déesse mère, etc. Il aurait peut-être été souhaitable d'attirer l'attention en note sur ces problématiques. Enfin, l'ouvrage aurait largement bénéficié d'une relecture attentive.

8 En conclusion, l'un des intérêts de cette étude est d'avoir confirmé à quel point les fonctions de protectrice de la cité, de divinité « poliade » forment un aspect essentiel de la figure d'Athéna. En revanche, si le lecteur pourra y puiser des informations utiles sur les cultes de la déesse dans cette région, il sera bien inspiré de considérer les interprétations proposées par l'A. avec prudence. 


\section{NOTES}

1. Voir à ce propos l'ouvrage édité par $\mathrm{Cl}$. PRÊTRE, Le donateur, l'offrande et la déesse. Systèmes votis dans les sanctuaires de déesses du monde grec. Actes du $31^{e}$ colloque international organisé par l'UMR Halma-Ipel (Université Charles-de-Gaulle/Lille 3, 13-15 décembre 2007), Liège, 2009 (Kernos, suppl. 23), conclusion p. 319-333.

2. Thèse de doctorat en préparation à l'Université de Liège sur «La vie religieuse dans l'île de Cos après le synécisme de 366 avant notre ère. Contribution à l'étude du fonctionnement du polythéisme grec ».

3. À Cos même, Chiron 31 (2001), p. 253-265, nº 1, 1. 22. Mais aussi ID 1513.

\section{AUTEURS}

STÉPHANIE PAUL

Université de Liège 